\title{
Using ethnotaxonomy to assess traditional knowledge and language vitality: a case study with the Vaie people of Sarawak, Malaysia
}

\begin{abstract}
This article demonstrates the potential of using ethnotaxonomy and nomenclature to assess the vitality status of indigenous languages and traditional knowledge at the ecosystem level. We collaborated with the Vaie people of Sarawak, Malaysia, applying a mixed methodology approach that relies on free-listing to a large extent. We applied the Traditional Knowledge and Language Vitality (TraLaVi) index to assess traditional knowledge and language vitality against five major parameters, specifically: language priority, retrieval of information, knowledge erosion, lexical recognition, and social support for exchange of traditional knowledge. The results show that with a TraLaVi score of 0.84 , the Vaie language can be considered "safe". Individuals practicing the traditional occupation of fishing fared better (mean=0.90) than those of the non-fishermen group (mean=0.77). However, when the language vitality was assessed using the Language Vitality and Endangerment assessment tool of UNESCO, the results indicate that the Vaie language could potentially be in the "unsafe" zone, highlighting the differences between the ecosystem based approach of TraLaVi and the macro-approach of UNESCO. However, these approaches can be applied in a complementary manner to generate a more accurate portrayal of the language vitality scenario.
\end{abstract}

Keyword: Folk names; Vernacular names; Language vitality and endangerment; Linguistic ethnobiology 